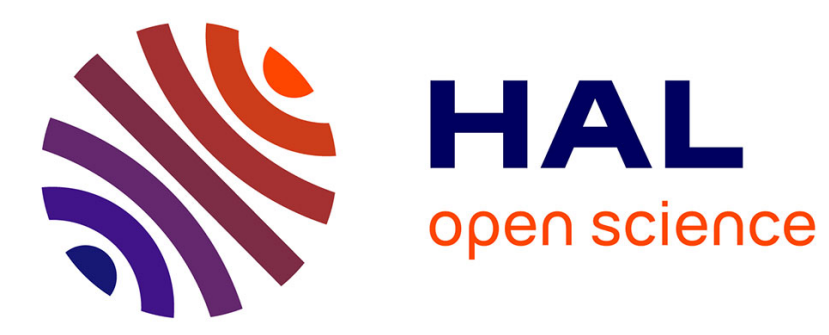

\title{
Microforce sensor for microbiological applications based on a floating-magnetic principle.
}

\author{
Ali Cherry, Joël Abadie, Emmanuel Piat
}

\section{To cite this version:}

Ali Cherry, Joël Abadie, Emmanuel Piat. Microforce sensor for microbiological applications based on a floating-magnetic principle.. IEEE International Conference on Robotics and Automation, ICRA'2007., Apr 2007, Rome, Italy. pp.1504-1509. hal-00162286

\section{HAL Id: hal-00162286 https://hal.science/hal-00162286}

Submitted on 13 Jul 2007

HAL is a multi-disciplinary open access archive for the deposit and dissemination of scientific research documents, whether they are published or not. The documents may come from teaching and research institutions in France or abroad, or from public or private research centers.
L'archive ouverte pluridisciplinaire HAL, est destinée au dépôt et à la diffusion de documents scientifiques de niveau recherche, publiés ou non, émanant des établissements d'enseignement et de recherche français ou étrangers, des laboratoires publics ou privés. 


\title{
Microforce sensor for microbiological applications based on a floating-magnetic principle
}

\author{
A. Cherry, J. Abadie and E. Piat
}

\begin{abstract}
In this paper, we present the design of a new magnetic nano and microforce sensor for microbiological applications. The sensing part of the sensor presents a naturally stable six degrees of freedom equilibrium state using the combination of upthrust buoyancy and magnetic force. The sensor allows force measurement without deformation of the sensing element using a feedback control loop and is able to measure the components, in the horizontal plan, of the external force applied. The measurement range varies between around $\pm 100 \mu N$ with a resolution of $20 n N$ and a linear output. The mechanical stiffness of the passive system is about $0.018 \mathrm{~N} . \mathrm{m}^{-1}$ (same order of magnitude than an AFM micro-cantilever). A complete static study and experimental validation of the used principle are presented in this paper.
\end{abstract}

\section{INTRODUCTION}

Recently, microscale sensing and manipulation have become a challenging issue mainly in micro-assembly of hybrid microsystems and biomanipulation. In fact, the potential applications are vast. In this context, many tools have been developped to visualize the micro world, but unfortunately it is not the same with regard to measurements of micro object mechanical parameters or concerning the forces applied on it.

In the field of single cell micromanipulation, the handling of individual cells is still often solved manually by human operators without force sensing. These tasks require long hours of practice and in spite of individual training, the failure rate remains very high. In fact, the high degree of accuracy needed for tools positioning and the weakness of the mechanical efforts involved in these tasks make them very difficult to achieve. It is important to notice that these forces are included in the micro Newton scale. Such small forces provide a difficult challenge for designing sensors that can output measurements with high resolution and high accuracy.

Such force measurements appear also useful in the field of the micro assembly. When manipulating micro objects, especially delicate structures, pure position control is usually not adequate or sufficient to ensure successful operation and prevent damage to the objects. Force control is often needed in order to achieve better manipulation results. Our objective is to improve the communication between the operator and the micro world by including new

This work was not supported by any organization

A. Cherry, J. Abadie and E. Piat are with Laboratoire d'Automatique de Besançon - UMR CNRS 6596 - ENSMM, France Emmanuel.piat@ens2m. fr ways of interaction such as for instance the possibility of feeling efforts.

Forces sensing is strongly related to the measurement of displacement or deformation of an elastic structure. The deformation is either detected by measuring the change in some material properties (electrical resistance, capacitance etc...), or directly measured by optical devices. The applied force is directly calculated using the structure stiffness which is established after calibration with a known force. A short selection of nano and microforce sensors based on this principle is described below.

A 3D micromanipulation system was developed by [1]. This system allows to control the interface forces in contacttype micromanipulations between objects and effector, using a multi-axial force sensor built with strain gauges. The force sensing is based on the piezoresistivity effect which has a good linearity in some conditions. This type of sensor must be calibrated before each measurement because of the plastic deformation. The size of the gauge must also be adapted to the sensing structure dimensions. In the case of microstructures, the gauge fabrication becomes difficult to achieve.

Sun introduced a design of a planar capacitive force sensor with 6 degrees of freedom used to characterize Mouse Zona Pellucida and quantify its mechanical property differences before and after fertilization [2]. This capacitive sensor is able to resolve normal forces applied to a cell as well as tangential forces generated by improperly aligned cell probes. However this design is fabricated using MEMS technology and can not measure constant forces.

Li designed a highly sensitive 1-D and 2-D sensor system for applications in micro-assembly and bio-manipulation using an in situ PVDF (polyvinylidene fluoride) piezoelectric sensor [3]. The sensor system has a resolution in the range of sub-micronewton. The charge developed across the plate is linearly proportional to the applied pressure and thickness but decrease with time when a non-varying load is applied.

Girot and Rougeot used a system based on atomic force microscope (AFM) to analyze the contact forces and remote forces at a microscopic scale within the framework of micromanipulation [4], [5]. The sensing element is a microcantilever with a stiffness $K=0.03 \mathrm{~N} / \mathrm{m}$. The applied force is proportional to the micro-cantilever deflection. The drawback of this technic is that it's a global quantification of force without indication of the measurement direction and thus the type of solicitation.

Other type of microforce sensors based on AFM used 
a carbon nanotube (CNT) probe, calibrated by the electromechanical resonance [6]. The CNT is attached to the AFM cantilever by an electron-beam-induced deposition (EBID). The sensor is able to measure the contact forces in piconewton order resolution by measuring the deformation of the CNT probe from FE-SEM images.

All the systems described before are monolithics. In the case of micromecanisms constituted by several assembled parts, friction prohibits correct force measurement in the microworld. One way to avoid friction problem, is to use levitation methods in these devices.

Our laboratory developed a microforce sensor based on magnetic levitation in entirely passive configuration using diamagnetic and magnetic materials [7], [8], [9]. The levitating part (see figures 1 and 2) is used as a one direction force sensing device. The displacement of this device, when an external force is applied on it, is measured using a laser beam and is proportional to this force. The absence of friction associated with a low stiffness $(K=0.021 \mathrm{~N} / \mathrm{m})$ makes the sensor highly sensitive. The only problem of this configuration is the weight limitation of the levitating part (less than 200 milligrams).

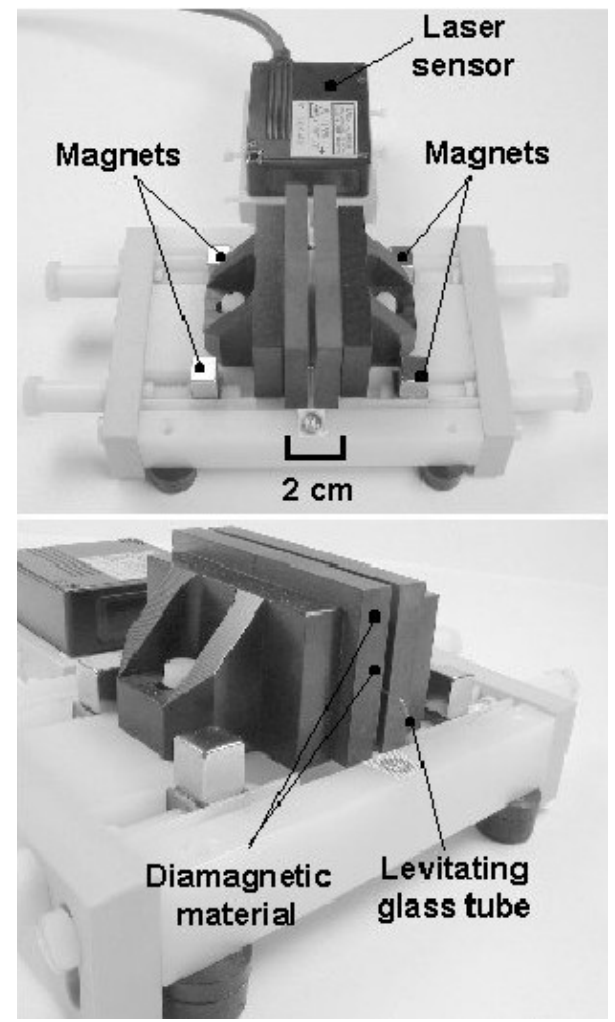

Fig. 1. Force sensor based on passive diamagnetic levitation

Because passive levitation is naturally stable and seems a very efficient and low cost principle to measure microforces, we decided to carry on the work initiated by [7] substituting diamagnetic levitation by a floating principle in order to suppress the weight limitation. The part used as the force sensing device is also naturally stable with 6 degrees of freedom (proof not included in this paper).

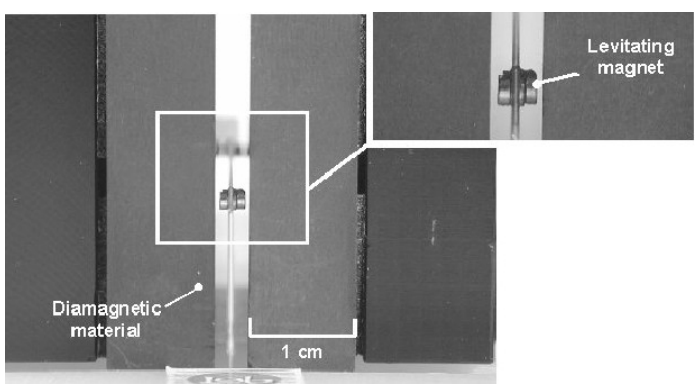

Fig. 2. Levitating force sensing device

This paper presents the description of this new micro forces sensor which is totally compatible with biological materials. Both static and dynamic studies are presented with a 1D model and model identification is explained. As this paper is focused mainly on the sensor design and not its complete 3D dynamic model, only a simplified 1D model is detailed here. Moreover, as the sensor allows measurements of forces without any displacement of the sensible part, we briefly present the design used to make possible the feedback control loop.

\section{SENSOR CONFIGURATION}

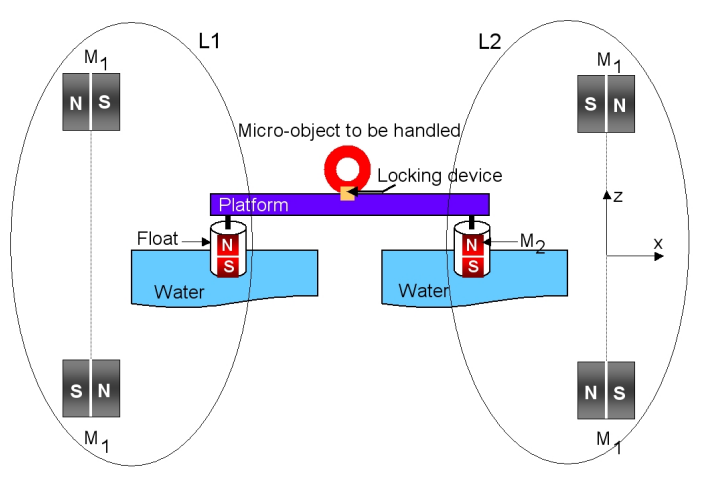

Fig. 3. Sensor configuration (two of the three buoyancy tanks are presented)

The sensing element is a triangular platform suspended by 3 small buoyancy tanks $L_{1}, L_{2}$ and $L_{3}$ and presents a naturally stable six degrees of freedom equilibrium state (see figure 3). Thus, the sensing device is the entire platform on which the object to be manipulated is locked. Each buoyancy tank is formed by 2 fixed cubic magnets $M_{1}$ $(5 \times 5 \mathrm{~mm})$ and a cylindrical moving magnet $M_{2}$ placed at the corner of the triangular platform, inside a float. The magnets $M_{1}$ are placed such that their north and south poles are in opposite directions. The distance between the 2 magnets $M_{1}$ influences the value of magnetic forces applied on the floating magnets $M_{2}$. The magnets $M_{1}$ and $M_{2}$ are made of $\mathrm{NdFeB}$ and the three floating magnets $M_{2}$ have a radius of $2 \mathrm{~mm}$ and a thickness of $2.5 \mathrm{~mm}$. The platform mass is supported against gravity by the combined upthrust buoyancy of the three floats. Thus, the platform weight is not an issue for this sensor. Magnetic forces of magnets $M_{1}$ provide stability of platform in the plane (xOy). The upthrust 
buoyancy provide the stability along $\vec{z}$ axis. The platform position is measured with three laser interferometers. In this case, an external force applied on the floating platform can be deduced according to its magnetic stiffness and displacement measured by the laser sensor.

In all next sections of this paper, the study will be only focused on one of three buoyancy tanks $L_{1}, L_{2}$ and $L_{3}$. The same study is also valid for the two other buoyancy tanks.

\section{STATIC STUDY}

In this section, the principle of force measurements according to $\vec{x}, \vec{y}$ and $\vec{z}$ directions is presented. Concerning the module $L_{1}$, at the equilibrium state position four external forces described below are applied on the floating magnet $M_{2}$ (see figure 4).

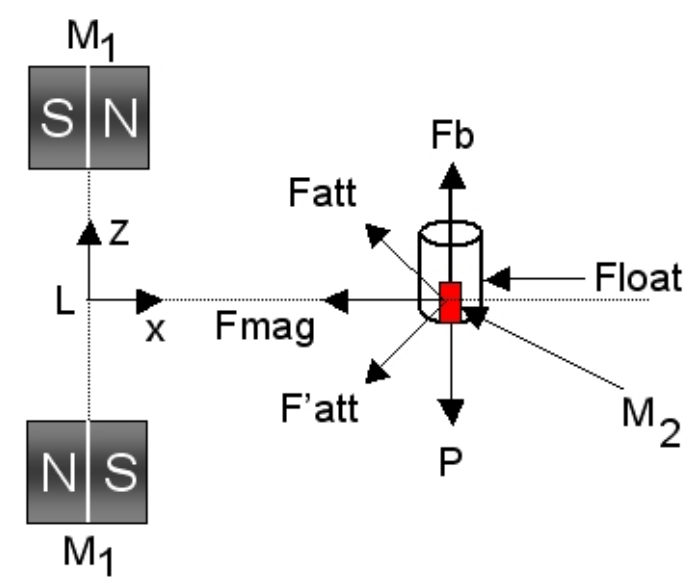

Fig. 4. Forces applied on the float

The top magnet $M_{1}$ applies an attractive force, called $\overrightarrow{F_{a t t}}$, on $M_{2}$. The lower magnet $M_{1}$ applies also an attractive force called $\overrightarrow{F_{a t t}^{\prime}}$ on $M_{2}$ obtained symmetrically to the plan $(x O y)$. We note $F_{m a g}$ the vectorial sum of $\overrightarrow{F_{a t t}}$ and $\overrightarrow{F_{a t t}^{\prime}}$. The third force is the upthrust buoyancy called $\vec{F}_{b}$ applied by the water at the centre of gravity $G$ of the float along $\vec{z}$. We will also suppose that $G$ is the centre of gravity of magnet $M_{2}$. The fourth force $\vec{P}$, also acting along $\vec{z}$, is the total weight of the float. When an unknown external force $\overrightarrow{F_{\text {ext }}}$ is applied at the centre of gravity $G$ of $M_{2}$, the new stable equilibrium state is obtained applying the principle of static at the float centre of gravity $G\left(x_{G}, y_{G}, z_{G}\right)$ :

$$
\overrightarrow{F_{m a g}}+\overrightarrow{F_{e x t}}+\vec{P}+\overrightarrow{F_{b}}=\overrightarrow{0}
$$

Because $\vec{F}_{b}$ and $\vec{P}$ are the largest forces (several orders of magnitude) compared to the other microforces, it is possible to consider that $\overrightarrow{F_{e x t}}$ is applied only in the $(x O y)$ plan. Thus, $\overrightarrow{F_{b}}$ compensates $\vec{P}$ and we can write $\overrightarrow{F_{\text {ext }}}=\overrightarrow{F_{\text {mag }}}$.

The determination of the external force $F_{\text {ext }}$ in the plane $(x O y)$ is conditioned by the determination of the magnetic force applied on $G$. The determination of $F_{m a g}$ is possible knowing the position of the floating magnet $M_{2}$.

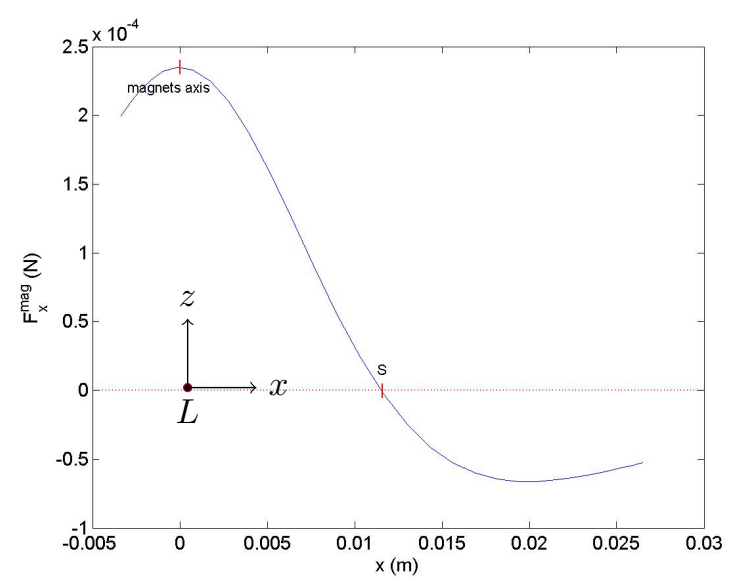

Fig. 5. Magnetic force produced by the 2 permanent magnets $M_{1}$ along $\vec{x}$

\section{A. Evaluation of $F_{\text {mag }}$}

The magnetic field produced at a point $M$ by a permanent magnet modelled by a surface distribution of loads is given by the following equation [10]:

$$
\begin{aligned}
\vec{B}_{\text {mag }}(M) & =\iint_{S^{+}} \frac{J}{4 \pi} \frac{A \vec{M}}{|A \vec{M}|} d S^{+} \\
& +\iint_{S^{-}} \frac{-J}{4 \pi} \frac{B \vec{M}}{|A \vec{M}|} d S^{-}
\end{aligned}
$$

The determination of the field components $B^{x}(M)$, $B^{y}(M)$ and $B^{z}(M)$ at the point $M$ can be done by the integration of the equation (2) along $x, y$ and $z$. The magnetic force applied on the magnet $M_{2}$ is given by the following equation :

$$
\vec{F}_{m a g}=\int_{V} \overrightarrow{m_{2}} \cdot \vec{\nabla} B_{m a g}(M) \cdot d v
$$

where $\overrightarrow{B_{\text {mag }}} \overrightarrow{ }(M)$ is the field produced by both magnets $M_{1}$ at a point $M$ inside the magnet $M_{2} . V$ is the volume of magnet $M_{2}$ and $\overrightarrow{m_{2}}$ its magnetization. This magnétisation is about $8.89 \times 10^{4} \mathrm{~A} \cdot \mathrm{m}^{-1}$.

Figure 5 presents the evaluation of the magnetic force along $x$ such that $F_{\text {mag }}^{\vec{y}}$ and $F_{\text {mag }}^{\vec{z}}$ equal to zero. This curve allowed us to calculate the magnetic stiffness $K_{m}^{x}=d F_{x} / d x$ of our passive floating system at any float position. On this curve we note a particular point $S$ which corresponds to the equilibrium of the float when $F_{\text {ext }}=0$. The cartesian coordinates of $S$ are $x=11.7 \mathrm{~mm}, y=0$ and $z=0$ according to a fixed reference axes $L(x, y, z)$. The origin $L$ of the fixed reference axes is located at the center of upper and lower magnets $M_{1}$ (see figure 4). One can notice that $\vec{m}_{2}$ does not have an influence on $S$ but only on $K_{m}^{x}$.

\section{Simplified MOdel of THE FLOAT (1D)}

\section{A. Dynamic equilibrium}

The dynamic equilibrium along $x$ is given by the following equation:

$$
F_{e x t}^{x}+F_{m a g}^{x}+F_{f}^{x}+F_{p}^{x}=m \ddot{x}
$$


in which $F_{e x t}^{x}$ is the external force applied on the floating magnet $M_{2}$ along $x$ and $F_{m a g}^{x}$ is the magnetic force produced by the two permanent magnets $M_{1}$. In case of small displacements around the point of equilibrium state $S$, this function is assumed linear and we can write :

$$
F_{m a g}^{\vec{x}}=K_{m}^{x} \cdot \vec{x}
$$

The stiffness $K_{m}^{x}$ at $S$ is about $18.9 \times 10^{-3} N . m^{-1}$.

$F_{f}^{x}$ is the viscous friction force between the float and water (atmosphere influence is neglected). Like for the magnetic force, in case of small displacements we can write :

$$
\overrightarrow{F_{f}^{x}}=K_{f}^{x} \cdot \overrightarrow{\dot{x}}
$$

in which $K_{f}^{x}$ is the friction coefficient.

Finally, $F_{p}^{x}$ is the total perturbation force, like capillary force (which disappears in case of symetric meniscus) and like the delayed return wave of water generated by the displacement of the float and reflected on the boards of the container.

According to a fixed reference axes with origin $S$, the equation (4) becomes :

$$
F_{e x t}^{x}+F_{p}^{x}=m \ddot{x}+K_{f}^{x} \dot{x}+K_{m}^{x} x
$$

\section{B. Parameters identification of $1 D$ model}

This section is devoted to the model parameters determination. Among these parameters, the magnetic stiffness $K_{m}^{x}$ is already identified knowing the magnetic force. The weight of the float $m$, measured with a microbalance is $5.66 \times 10^{-4}$ $k g$. The friction coefficient $K_{f}^{x}$, is evaluated using the free response of our system. It is obtained by pushing manually the float beyond the equilibrium point $S$ and rapidly releasing it. These measurements were done using a laser displacement sensor which has $1 \mu \mathrm{m}$ resolution (see figure 6).

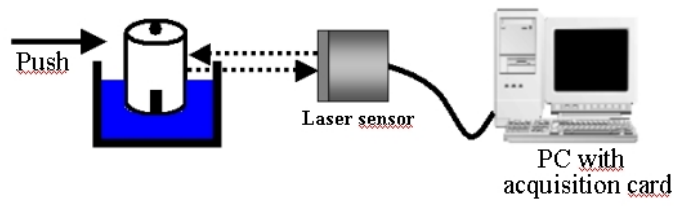

Fig. 6. Experimental measurements of the free response

The figure 7 shows the response of the experimental system and the simulation curve after the evaluation of the friction coefficient $K_{f}^{x}$ (this coefficient has been set so that both curves are the closest possible). This friction coefficient is $0.8 \times 10^{-3} \mathrm{~N} \cdot \mathrm{s} \cdot \mathrm{m}^{-1}$. The difference visible on the graph between the simulated and experimental curves is due to the 1-D model which doesn't represent correctly the 2D magnetic coupling on $x$ and $y$ axes. Perturbation forces explained before could also possibly have an unknown influence, which has to be investigated.

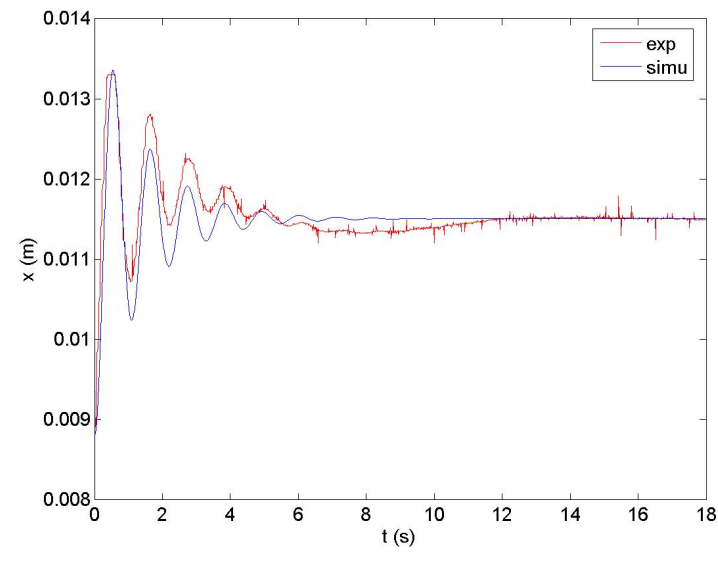

Fig. 7. float response along $\vec{x}$

\section{FORCE MEASUREMENT WITHOUT DISPLACEMENT}

We saw previously that force sensing is closely related to the measurement of the sensitive part displacement. This displacement can generate drawbacks in the case of high precision tasks such as biological cells micromanipulation or micro-assembling. For instance, the force sensitive part on figure 3 is the entire plateform. If this plateform moves when external forces are apply on the micro object, the microobject will also move, which can be problematic for the operator. Thus, the key idea here is to developp a device wich allows force measurements without any (or only very small) displacements of the sensitive part, thanks to an active control. The design used to make possible the feedback control loop is composed of two coils placed on both sides, above and below the floating magnet (see figure 8).

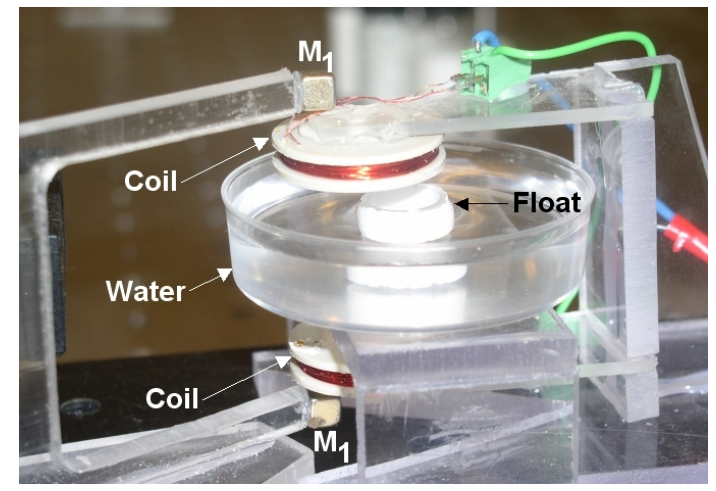

Fig. 8. Floating mechanism $L_{1}$ equipped with two coils

In case of an external force applied to the float which tends to push it beyond the point $S$, the activation of coils will produce an opposed electromagnetic force $\overrightarrow{F_{\text {elec }}} \overrightarrow{\text { that will }}$ maintain the float on $S$. In this case, the current $I$ in the coils is the new physical value related to the external force.

Figure 9 shows the step response for positive and negative current $I$. The displacement $x$ obtained is the combination of both magnetic and electromagnetic behavior due to $\overrightarrow{F_{m a g}}$ and $\overrightarrow{F_{\text {elec }}}$. The response for positive and negative current is 


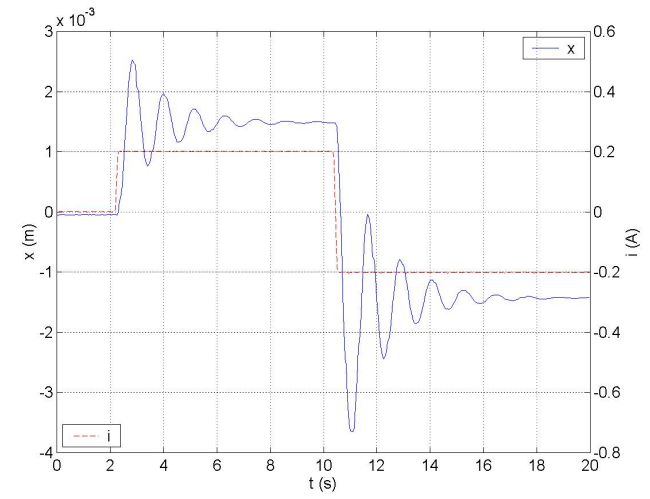

Fig. 9. Step response for positive and negative current

not the same because $\overrightarrow{F_{m a g}}$ is not symmetrical around $S$.

\section{A. Evaluation of $F_{\text {elec }}$}

The magnetic field produced by a coil at a point $M$ (in a cylindrical axes reference) is given by the following equations [11]:

$$
B_{\text {elec }}(M)=\left\{\begin{array}{l}
B^{r}(M)=\frac{\mu_{0}}{2 \pi}\left(I \pi N a^{2}\right) \frac{\cos \theta}{r^{3}} \\
B^{\theta}(M)=\frac{\mu_{0}}{2 \pi}\left(I \pi N a^{2}\right) \frac{\sin \theta}{r^{3}} \\
B^{\phi}(M)=0
\end{array}\right.
$$

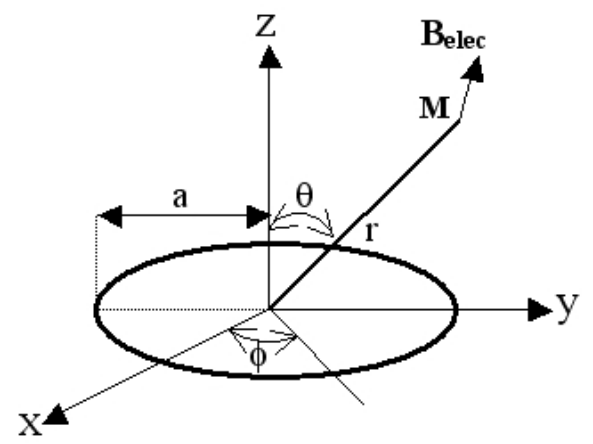

Fig. 10. Circular spire

in which : r, $\theta$ and $\phi$ are the components of the cylindrical coordinates (see figure 10), $N$ is the number of spires, $a$ is the radius of the coil and $I$ the current. These equations are valid in the case of microcoils, supposing that all spires are concentrated in the same place.

Like for magnetic force, the electromagnetic force applied to the magnet $M_{2}$ centre of gravity $G$ is given by the following equation :

$$
\vec{F}_{\text {elec }}=\int_{V} \overrightarrow{m_{2}} \cdot \vec{\nabla} B_{\text {elec }}(M) \cdot d v
$$

The new dynamic equilibrium along $x$ in the presence of the two coils is given by the following equation:

$$
F_{\text {ext }}^{x}+F_{\text {elec }}^{x}+F_{p}^{x}=m \ddot{x}+K_{f}^{x} x+x K_{m}^{x}
$$

In this equation the electromagnetic force of two coils along $x$ axis $\left(F_{\text {elec }}^{x}\right)$ has been introduced to the equation (4).

\section{B. Experimental validation}

Thanks to the magnetic field equations of coil, We have built a complete analytical model programmed in $\mathrm{C}++$ and embedded in Matlab/Simulink. This model allowed us to simulate the electromagnetic force $\vec{F}_{\text {elec }}$ applied to the float. The figure 11 presents the evolution of this force along $x$ in the reference axes $(L, \vec{x}, \vec{z})$. The coils axis (point $B$ ) is $5.7 \mathrm{~mm}$ far from $L$. The coil used for this simulation is a circular coil of 50 spires with a radius of $1 \mathrm{~cm}$ and a maximum current of $0.5 \mathrm{~A}$. The maximum value of the electromagnetic force is produced $6 \mathrm{~mm}$ far from the coils axis. This value is about $68 \mu N$ for a current $I$ equal to 0.4 A.

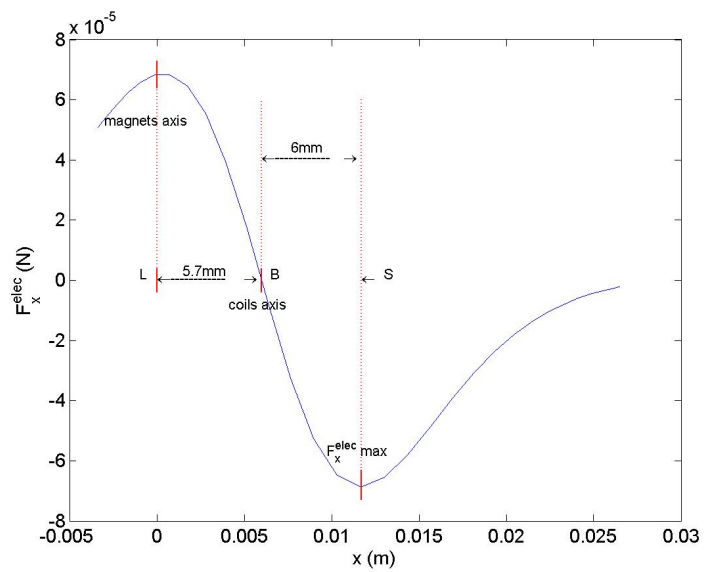

Fig. 11. Electomagnetic force produced by the two coils

The distance $L B$ is choosen in order to have a maximum electromagnetic force at $S$ (see figure 12) which gives the sensor usefull range.

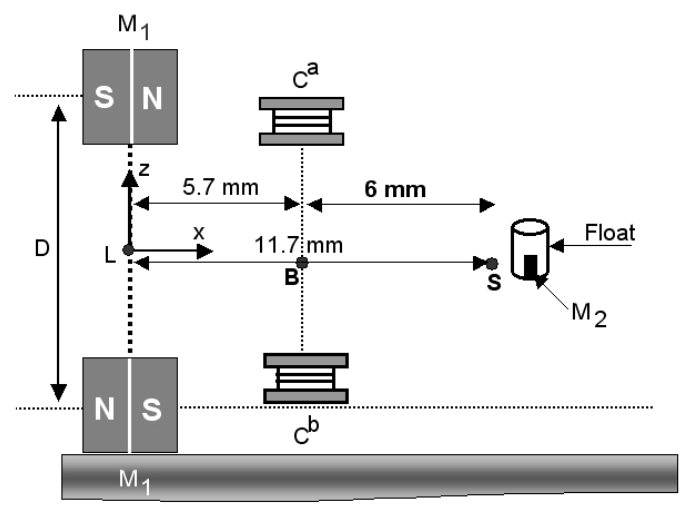

Fig. 12. Optimal position of coils

The figure 13 represents the evaluation of the sum of magnetic and electromagnetic forces along $x$ for different values of $I$. In the final design of the sensor, this sum will have to be controlled for each float in order to maintain the 
platform on $S$. We can notice that when $I$ is equal to zero, we have a null electromagnetic force and the curve drawn with little stars represents the magnetic force only, like on figure 5. At the point $S$ of static equilibrium, the magnetic force is always null. On this point the maximum external force which can be measured by the device, without platform displacement, will be equal to the maximum electromagnetic force that can be generated at this point.

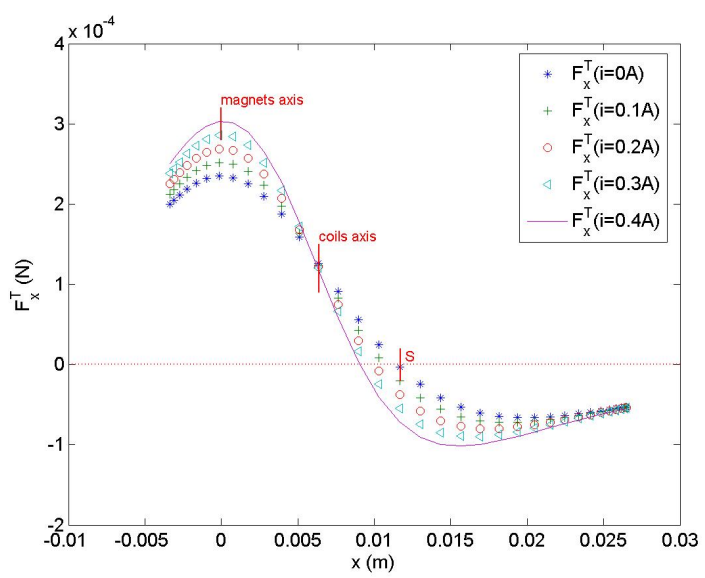

Fig. 13. Evaluation of the total force along $\vec{x}$ for different $I$

To calculate the electrical stiffness of our configuration, we have also plot the electromagnetic force $\vec{F}_{\text {elec }}^{x}$ when the float is positionned on the point of stable equilibrium $S$ (thus $F_{m a g}=0$ ), for different values of $I$ (see figure 14).

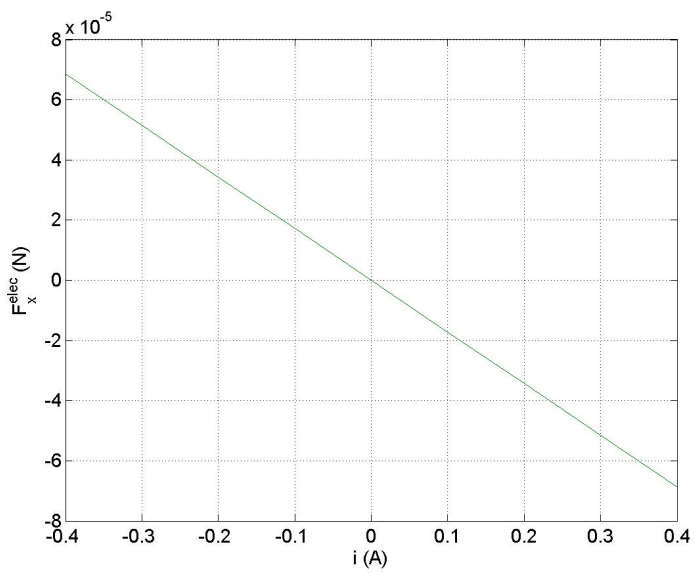

Fig. 14. Electromagnetic force $\vec{F}_{\text {elec }}^{x}$ at $S$ for different values of $I$

We can see easily that the relation between $I$ and the electromagnetic force $\vec{F}_{\text {elec }}^{x}$ is linear, that allowed us to write:

$$
\vec{F}_{\text {elec }}^{x}=K_{e}^{x} \cdot i(t)
$$

Where $K_{e}^{x}$ is the electrical stiffness. It's value is $1.716 \times$ $10^{-4}$ N. $A^{-1}$.

\section{Conclusion}

A six degrees of freedom microforce sensor prototype was presented in this paper. The sensing part is a floating platform stabilized by a magnetic field. Compared to our previous force sensor using diamagnetic levitation this configuration hasn't weight limitation of the levitating sensing part. The sensor allows force measurements without displacement of the platform. The current $I$ in the coils, proportional to the external force $F^{e x t}$, provide a linear output. The global platform mechanical stiffness $K_{m}$ is about $18.3 \times 10^{-3}$ $N . m^{-1}$ (close to the stiffness of a classic AFM microcantilever). In this paper we have also presented a complete analytical model of one of the three buoyancy tanks. This model allowed us to simulate the electromagnetic force $F^{\text {elec }}$ applied to the float by the coils and the evaluation of both magnetic and electromagnetic forces for different values of $I$. The study of the frequency response, the perturbation forces rejection, the platform $3 D$ modeling, the position control, the assembling and experimentation of the complete sensor is currently under development. Concerning the frequency response, this one will certainly exhibit modal resonance because of the delayed return wave of water generated by the displacement of the float.

\section{REFERENCES}

[1] F. Arai, A. Kawaji, T. Sugiyama, Y. Onomura, M. Ogawa, T. Fukuda, $\mathrm{H}$. Iwata, and K. Itoigawa, "3d micromanipulation system under microscope," International symposium on micromechatronics and human science, pp. 127-134, 1998.

[2] Y. Sun, W. Kai-Tak, K. Roberts, J. Bischof, and J. Bradley, "Mechanical property characterization of mouse zona pellucida," IEEE transactions on nanobioscience, vol. 2, no. 4, pp. 279-285, December 2003.

[3] W. Li and N. Xi, "Novel micro gripping, probing and sensing devices for single-cell surgery," Proceedings of the 26th international conference of the IEEE EMBS San Francisco, CA, USA, pp. 25912594, September 2004.

[4] P. Rougeot, S. Régnier, and N. Chaillet, "Forces analysis for micromanipulation," Proceedings 2005 IEEE international symposium on computational intelligence in robotics and automation, espoo, Finland, pp. 105-110, june 2005 .

[5] M. Girot, M. Boukhalel, and S. Régnier, "Towards a non-destructive in vitro biomechanical characterization," ASM - IEEE EMBS Conference on Bio-, Micro- and Nanosystems, San Francisco, USA, janvier 2006.

[6] F. Arai, M. Nakajima, L. Dong, and T. Fukuda, "Pico-newton order force measurement using a calibrated carbon nanotube probe by electrmechanical resonance," Proceedings of the 2003 IEEE international conference on robotics and automation Taipei, Taiwan, pp. 300-305, september 14-19 2003.

[7] M. Boukallel, E. Piat, and J. Abadie, "Passive diamagnetic levitation: theoretical foundations and application to the design of a micro-nano force sensor," Proceedings of the 2003 IEEE/RSJ intl. Conference on Intelligent Robots and Systems Las Vegas, Nevada, pp. 1062-1067, October 2003.

[8] M. Boukallel, M. Gauthier, E. Piat, J. Abadie, and C. Roux, "Microrobots for in vitro fertilization applications," Journal of American society - Cellular and Molecular biology, vol. 50, no. 3, may 2004.

[9] M. Boukallel, J. Abadie, and E. Piat, "Levitated micro-nano force sensor using diamagnetic levitation," Proc. of the IEEE International Conference of Robotics and Automation, pp. 3219-3224, september 2003.

[10] F. Bancel and G. Lemarquant, "Three-dimensional analytical optimization of permanent magnets alternated structure," IEEE Transactions on Magnetics, vol. 34(1), pp. 242-247, 1998.

[11] J. D. Jackson, Classical Electrodynamics, T. Edition, Ed. John Willey and sons, 1998. 